TRANSACTIONS OF THE

AMERICAN MATHEMATICAL SOCIETY

Volume 357, Number 5, Pages 1915-1929

S 0002-9947(04)03681-5

Article electronically published on September 2, 2004

\title{
BLOW-UP EXAMPLES FOR SECOND ORDER ELLIPTIC PDES OF CRITICAL SOBOLEV GROWTH
}

\author{
OLIVIER DRUET AND EMMANUEL HEBEY
}

\begin{abstract}
Let $(M, g)$ be a smooth compact Riemannian manifold of dimension $n \geq 3$, and $\Delta_{g}=-\operatorname{div}_{g} \nabla$ be the Laplace-Beltrami operator. Let also $2^{\star}$ be the critical Sobolev exponent for the embedding of the Sobolev space $H_{1}^{2}(M)$ into Lebesgue's spaces, and $h$ be a smooth function on $M$. Elliptic equations of critical Sobolev growth such as
\end{abstract}

$$
\Delta_{g} u+h u=u^{2^{\star}-1}
$$

have been the target of investigation for decades. A very nice $H_{1}^{2}$-theory for the asymptotic behaviour of solutions of such equations has been available since the 1980's. The $C^{0}$-theory was recently developed by Druet-HebeyRobert. Such a theory provides sharp pointwise estimates for the asymptotic behaviour of solutions of $(E)$. It was used as a key point by Druet to prove compactness results for equations such as $(E)$. An important issue in the field of blow-up analysis, in particular with respect to previous work by Druet and Druet-Hebey-Robert, is to get explicit nontrivial examples of blowing-up sequences of solutions of $(E)$. We present such examples in this article.

Let $(M, g)$ be a smooth compact Riemannian manifold of dimension $n \geq 3$. We denote by $H_{1}^{2}(M)$ the standard Sobolev space of functions in $L^{2}$ with one derivative in $L^{2}$. We let $\left(h_{\varepsilon}\right)$ be a sequence of $C^{0, \theta}$ functions on $M, 0<\theta<1$, and consider equations such as

$$
\Delta_{g} u+h_{\varepsilon} u=u^{2^{\star}-1}
$$

where $\Delta_{g}=-d i v_{g} \nabla$ is the Laplace-Beltrami operator, $2^{\star}=2 n /(n-2)$ is the critical Sobolev exponent for the embedding of the Sobolev space $H_{1}^{2}(M)$ into Lebesgue's spaces, and $u$ is required to be positive. We let $\left(u_{\varepsilon}\right)$ be a bounded sequence in $H_{1}^{2}(M)$ of solutions of $\left(E_{\varepsilon}\right)$ in the sense that for any $\varepsilon$,

$$
\Delta_{g} u_{\varepsilon}+h_{\varepsilon} u_{\varepsilon}=u_{\varepsilon}^{2^{\star}-1}
$$

and $\left\|u_{\varepsilon}\right\|_{H_{1}^{2}} \leq \Lambda$ where $\Lambda>0$ is independent of $\varepsilon$. We also assume that the $h_{\varepsilon}$ 's are uniformly bounded and that they converge in $L^{2}$ to some limiting function $h_{0}$. Then we know, since the 1980's, how to describe the $H_{1}^{2}$-asymptotic behaviour of the $u_{\varepsilon}$ 's as $\varepsilon \rightarrow 0$. Such a description when dealing with equations like $\left(E_{\varepsilon}\right)$ was proved in Struwe [17. Related references are Brézis-Coron 1, 2, Lions [12, SacksUhlenbeck [14, Schoen [15], and Wente 18]. In particular, following [17, we do

Received by the editors September 5, 2003.

2000 Mathematics Subject Classification. Primary 58E35.

(C)2004 American Mathematical Society 
get that, up to a subsequence,

$$
u_{\varepsilon}=u_{0}+\sum_{i=1}^{k} B_{\varepsilon}^{i}+R_{\varepsilon}
$$

and

$$
\left\|u_{\varepsilon}\right\|_{H_{1}^{2}}^{2}=\left\|u_{0}\right\|_{H_{1}^{2}}^{2}+\sum_{i=1}^{k}\left\|B_{\varepsilon}^{i}\right\|_{H_{1}^{2}}^{2}+r_{\varepsilon}
$$

where $u_{0}$ is a solution of the limit equation

$$
\Delta_{g} u+h_{0} u=u^{2^{\star}-1}
$$

where $k$ is some nonnegative integer, $\|\cdot\|_{H_{1}^{2}}$ is the standard norm on $H_{1}^{2}(M)$, the $B_{i, \varepsilon}$ 's are standard bubbles, $r_{\varepsilon} \rightarrow 0$ as $\varepsilon \rightarrow 0$, and $R_{\varepsilon} \rightarrow 0$ in $H_{1}^{2}(M)$ as $\varepsilon \rightarrow 0$.

Standard bubbles are rescalings of fundamental positive solutions of the Euclidean equation $\Delta u=u^{2^{\star}-1}$. Such solutions have been classified by CaffarelliGidas-Spruck [3]. We refer also to Obata [13. More precisely, a standard bubble is a sequence $\left(B_{\varepsilon}\right)$ of functions given by

$$
B_{\varepsilon}(x)=\left(\frac{\mu_{\varepsilon}}{\mu_{\varepsilon}^{2}+\frac{d_{g}\left(x_{\varepsilon}, x\right)^{2}}{n(n-2)}}\right)^{\frac{n-2}{2}},
$$

where $\left(x_{\varepsilon}\right)$ is a converging sequence of points in $M$, and $\left(\mu_{\varepsilon}\right)$ is a sequence of positive real numbers such that $\mu_{\varepsilon} \rightarrow 0$ as $\varepsilon \rightarrow 0$. The $x_{\varepsilon}$ 's are referred to as the centers of the bubble, and the $\mu_{\varepsilon}$ 's as the weights of the bubble. Clearly, $B_{\varepsilon} \rightarrow 0$ in $C_{l o c}^{0}\left(M \backslash\left\{x_{0}\right\}\right)$ as $\varepsilon \rightarrow 0$, where $x_{0}$ is the limit of the $x_{\varepsilon}$ 's, while $B_{\varepsilon}\left(x_{\varepsilon}\right) \rightarrow+\infty$ as $\varepsilon \rightarrow 0$. Moreover, while $B_{\varepsilon} \rightarrow 0$ in $L^{2}$ as $\varepsilon \rightarrow 0$, we do have that

$$
\left\|B_{\varepsilon}\right\|_{H_{1}^{2}}^{2}=K_{n}^{-n}+r_{\varepsilon}
$$

for all $\varepsilon$, where $r_{\varepsilon} \rightarrow 0$ as $\varepsilon \rightarrow 0$ and $K_{n}$, explicitly known and depending only on $n$, is the sharp constant $K$ for the Euclidean Sobolev inequality $\|u\|_{2^{\star}} \leq K\|\nabla u\|_{2}$. Independently, it is easily seen that a decomposition like (0.1)-(0.2) is unique in the following sense: $(0.1)-(0.2)$ hold with respect to two sets $\left(B_{\varepsilon}^{i}\right)$ and $\left(\tilde{B}_{\varepsilon}^{i}\right)$ of bubbles, $i=1, \ldots, k$, if and only if, up to renumbering,

$$
\lim _{\varepsilon \rightarrow 0} \frac{\tilde{\mu}_{\varepsilon}^{i}}{\mu_{\varepsilon}^{i}}=1 \quad \text { and } \quad \lim _{\varepsilon \rightarrow 0} \frac{d_{g}\left(x_{\varepsilon}^{i}, \tilde{x}_{\varepsilon}^{i}\right)}{\mu_{\varepsilon}^{i}}=0
$$

for all $i=1, \ldots, k$, where the $\left(B_{\varepsilon}^{i}\right)$ 's and $\left(\tilde{B}_{\varepsilon}^{i}\right)$ 's are respectively defined with respect to $\left(x_{\varepsilon}^{i}\right),\left(\mu_{\varepsilon}^{i}\right)$ and $\left(\tilde{x}_{\varepsilon}^{i}\right),\left(\tilde{\mu}_{\varepsilon}^{i}\right)$. A possible reference of introductory nature on the subject is Hebey [9].

The decomposition (0.1)-(0.2) provides what we refer to as the $H_{1}^{2}$-theory for blow-up. The $C^{0}$-theory was recently developed in Druet-Hebey-Robert [6, 7]. It is proved in Druet-Hebey-Robert [7] that if the convergence of $h_{\varepsilon}$ to $h_{0}$ is in $C^{0, \theta}(M)$, $\theta \in(0,1)$, then, in addition to (0.1)-(0.2), there also exists $C>1$ such that, up to 
a subsequence,

$$
\begin{aligned}
& \left(1-\eta_{\varepsilon}\right) u_{0}(x)+\frac{1}{C} \sum_{i=1}^{k} B_{\varepsilon}^{i}(x) \\
& \quad \leq u_{\varepsilon}(x) \leq\left(1+\eta_{\varepsilon}\right) u_{0}(x)+C \sum_{i=1}^{k} B_{\varepsilon}^{i}(x)
\end{aligned}
$$

for all $\varepsilon$ and all $x$, where the $\eta_{\varepsilon}$ 's are positive real numbers, independent of $x$, satisfying that $\eta_{\varepsilon} \rightarrow 0$ as $\varepsilon \rightarrow 0$. Moreover (see again [6, 7]) the constant $C$ can be chosen as close as we want to 1 in small neighbourhoods of the geometrical points, defined as the limits of the centers of the bubbles. In particular, the exact $C^{0}$ asymptotic behaviour of the $u_{\varepsilon}$ 's is obtained. This provides a complete description of the blow-up at the $C^{0}$-level. Such a description was then used as a key point by Druet [4] when proving compactness results for equations such as $\left(E_{\varepsilon}\right)$ in the spirit of those obtained by Schoen [15, 16]. As a remark, it is not possible to develop a general $C^{0}$-theory for which $R_{\varepsilon}$ in $(0.1)$ would satisfy that $R_{\varepsilon} \rightarrow 0$ in $C^{0}(M)$ as $\varepsilon \rightarrow 0$.

Quite surprisingly, open questions, in particular with respect to the works developed in Druet 4 and Druet-Hebey-Robert 6, 7], are to know whether or not there exist examples of $h_{\varepsilon}$ 's and $u_{\varepsilon}$ 's such that in $(0.1)-(0.2)$

(Q1) $k \geq 1$, and $u_{0}(x) \neq 0$ for all $x$, or such that

(Q2) $k \geq 2$, and $d_{g}\left(x_{\varepsilon}^{i}, x_{\varepsilon}^{j}\right) \rightarrow 0$ as $\varepsilon \rightarrow 0$, where the $B_{\varepsilon}^{i}$ 's in the decomposition (0.1)-(0.2) are given by (0.3) with $x_{\varepsilon}=x_{\varepsilon}^{i}$ and $\mu_{\varepsilon}=\mu_{\varepsilon}^{i}$. As an interesting feature on (Q1), Druet [4] proved that if the convergence of $h_{\varepsilon}$ to $h_{0}$ is in $C^{2}$, and $n=3,4,5$, then $u_{0}(x)=0$ for all $x$. Theorem 0.1 below indicates that this is not anymore the case when $n \geq 6$. A positive answer to (Q2), as given by Theorem 0.1 below, shows that bubbles in the decomposition (0.1)-(0.2) associated to sequences of solutions of equations such as $\left(E_{\varepsilon}\right)$ are not necessarily isolated. This result is false in dimension 3 where bubbles have to be isolated (see Li-Zhu [1]). Independently, thanks to Druet 4], we know that when $n \neq 6$, if the $h_{\varepsilon}$ 's and $u_{\varepsilon}$ 's are such that $k \geq 1$ in $(0.1)-(0.2)$, and the convergence of $h_{\varepsilon}$ to $h_{0}$ is in $C^{2}$, then, necessarily, there exists at least one $x \in M$ such that

$$
h_{0}(x)=\frac{n-2}{4(n-1)} S_{g}(x),
$$

where $S_{g}$ is the scalar curvature of $g$. A more precise statement is in Druet [4]. Assuming that the convergence of $h_{\varepsilon}$ to $h_{0}$ does not hold in $C^{2}(M)$, we also address the question to know whether or not there exist examples of $h_{\varepsilon}$ 's and $u_{\varepsilon}$ 's such that

(Q3) $k \geq 1$, and $h_{0}(x) \neq \frac{n-2}{4(n-1)} S_{g}(x)$ for all $x$, where $S_{g}$ is the scalar curvature of $g$. A more pertinent question is whether or not (especially when $n=6$ ) we can ask that

(Q4) $k \geq 1, h_{\varepsilon}(x) \geq \frac{n-2}{4(n-1)} S_{g}(x)+\varepsilon_{0}$, and $h_{0}(x) \geq \frac{n-2}{4(n-1)} S_{g}(x)+\varepsilon_{0}$ for all $x$, where $\varepsilon_{0}>0$, and $S_{g}$ is the scalar curvature of $g$.

We answer questions (Q1)-(Q4) in this article. The examples we provide involve easy mathematics. We let $\left(S^{n}, h\right)$ be the unit $n$-sphere. The scalar curvature of $h$ 
is $S_{h}=n(n-1)$. We let $\Phi_{0}$ be the constant function

$$
\Phi_{0} \equiv \frac{n(n-2)}{4}
$$

and consider, as above, equations such as

$$
\Delta_{h} u+h_{\varepsilon} u=u^{2^{\star}-1} .
$$

When $h_{\varepsilon}=\Phi_{0},(0.4)$ is the Yamabe equation on the unit $n$-sphere. If $\left(u_{\varepsilon}\right)$ is a sequence of smooth positive solutions of $(0.4)$ we then have that

$$
\Delta_{h} u_{\varepsilon}+h_{\varepsilon} u_{\varepsilon}=u_{\varepsilon}^{2^{\star}-1} .
$$

Assuming that the $h_{\varepsilon}$ 's are bounded in $L^{\infty}\left(S^{n}\right)$, and that they converge in $L^{2}\left(S^{n}\right)$ to some continuous function $h_{0}$ as $\varepsilon \rightarrow 0$, decomposition (0.1)-(0.2) holds. We say that the $u_{\varepsilon}$ 's blow up as $\varepsilon \rightarrow 0$ if $k \geq 1$ in (0.1)-(0.2). Our first result is as follows:

Theorem 0.1. Let $\left(S^{n}, h\right)$ be the unit $n$-sphere, $n \geq 6$. Then

(T1) there exist examples of $h_{\varepsilon}$ 's and $u_{\varepsilon}$ 's satisfying (0.5) such that the sequence $\left(h_{\varepsilon}\right)$ is bounded in $L^{\infty}\left(S^{n}\right)$, the sequence $\left(h_{\varepsilon}\right)$ converges to $h_{0}=\Phi_{0}$ in $L^{p}\left(S^{n}\right)$ for all $p \geq 1$, the sequence $\left(u_{\varepsilon}\right)$ blows up (so that $k \geq 1$ ), and $u_{0}(x) \neq 0$ for all $x$.

(T2) there exist examples of $h_{\varepsilon}$ 's and $u_{\varepsilon}$ 's satisfying $(0.5)$ such that the sequence $\left(h_{\varepsilon}\right)$ converges to $h_{0}=\Phi_{0}$ in $C^{1}\left(S^{n}\right)$, the sequence $\left(u_{\varepsilon}\right)$ blows up with two bubbles (so that $k \geq 2$ ), and the distances between the centers of the two bubbles go to 0 as $\varepsilon \rightarrow 0$.

(T3) there exist examples of $h_{\varepsilon}$ 's and $u_{\varepsilon}$ 's satisfying (0.5) such that the sequence $\left(h_{\varepsilon}\right)$ is bounded in $L^{\infty}\left(S^{n}\right)$, the sequence $\left(h_{\varepsilon}\right)$ converges to some continuous function $h_{0}$ in $L^{p}\left(S^{n}\right)$ for all $p \geq 1$, the sequence $\left(u_{\varepsilon}\right)$ blows up (so that $k \geq 1$ ), and $h_{0}(x) \neq \Phi_{0}(x)$ for all $x$.

When $n=6$, there exist examples of $h_{\varepsilon}$ 's and $u_{\varepsilon}$ 's satisfying $(0.5)$ such that the sequence $\left(h_{\varepsilon}\right)$ is bounded in $L^{\infty}\left(S^{n}\right)$, the sequence $\left(h_{\varepsilon}\right)$ converges to some continuous function $h_{0}$ in $L^{p}\left(S^{n}\right)$ for all $p \geq 1$, such that $h_{\varepsilon}$ and $h_{0}$ satisfy that

$$
h_{\varepsilon} \geq \Phi_{0}+\varepsilon_{0} \text { and } h_{0} \geq \Phi_{0}+\varepsilon_{0}
$$

for all $\varepsilon>0$ and some $\varepsilon_{0}>0$, and such that the sequence $\left(u_{\varepsilon}\right)$ blows up (so that $k \geq 1$ ). In particular, when $n \geq 6$, questions (Q1)-(Q3) receive positive answers, and when $n=6$, question (Q4) also receives a positive answer.

We know since the work of Schoen [15, 16] (see also Li-Zhang [10] and Li-Zhu 11]) that there is an a priori bound for the energy of solutions of the Yamabe equation. We define here the energy $E(u)$ of a function $u \in H_{1}^{2}$ by $E(u)=\|u\|_{2^{\star}}$. Another question of interest, in particular with respect to the works developed in Druet [4] and Druet-Hebey-Robert [6, 7, is whether or not there exists such a bound for solutions of equations such as $\left(E_{\varepsilon}\right)$. The following result hopefully answers this question in the negative.

Theorem 0.2. Let $\left(S^{n}, h\right)$ be the unit $n$-sphere, $n \geq 6$. Then there exist examples of $h_{\varepsilon}$ 's and $u_{\varepsilon}$ 's satisfying $(0.5)$ such that the sequence $\left(h_{\varepsilon}\right)$ converges to $h_{0}=\Phi_{0}$ in $C^{1}\left(S^{n}\right)$ and such that the energies $E\left(u_{\varepsilon}\right)$ of the $u_{\varepsilon}$ 's satisfy that $E\left(u_{\varepsilon}\right) \rightarrow+\infty$ as $\varepsilon \rightarrow 0$.

An interesting feature in Theorem 0.2 is that the limit equation is the Yamabe equation for which, as already mentioned, we do have a priori bounds on the energy. In this specific case of the unit sphere, only one level is involved and the positive 
solutions of the Yamabe equation are known. We prove Theorem 0.1 in section 1. Strong interactions are discussed at the end of the section. We prove Theorem 0.2 in section 2 .

\section{Proof of Theorem 0.1}

We prove Theorem 0.1 in what follows. We assume $n \geq 6$. Given $\varepsilon>0$, and $i=1,2$, we let $\left(x_{i, \varepsilon}\right) \in S^{n}$ be two sequences of points in $S^{n}$, and $\left(\beta_{i, \varepsilon}\right) \in \mathbb{R}$ be two sequences of real numbers such that $\beta_{i, \varepsilon}>1$ for all $i=1,2$ and all $\varepsilon>0$. Given $\lambda>0$, we let

$$
u_{\varepsilon}=\lambda B_{1, \varepsilon}+B_{2, \varepsilon}
$$

where

$$
B_{i, \varepsilon}(x)=\left(\frac{n(n-2)}{4}\left(\beta_{i, \varepsilon}^{2}-1\right)\right)^{\frac{n-2}{4}}\left(\beta_{i, \varepsilon}-\cos d_{h}\left(x_{i, \varepsilon}, x\right)\right)^{1-\frac{n}{2}} .
$$

For $i=1,2$, and any $\varepsilon>0$,

$$
\Delta_{h} B_{i, \varepsilon}+\frac{n(n-2)}{4} B_{i, \varepsilon}=B_{i, \varepsilon}^{2^{\star}-1}
$$

where $2^{\star}=2 n /(n-2)$ is the critical Sobolev exponent. Clearly, $u_{\varepsilon} \in C^{\infty}\left(S^{n}\right)$ and $u_{\varepsilon}>0$ for all $\varepsilon>0$. An easy claim is that

$$
\Delta_{h} u_{\varepsilon}+h_{\varepsilon} u_{\varepsilon}=u_{\varepsilon}^{2^{\star}-1}
$$

where the linear term $h_{\varepsilon}$ is given by

$$
h_{\varepsilon}=\Phi_{0}+\Phi_{\varepsilon}
$$

and

$$
\Phi_{\varepsilon}=\frac{\left(\lambda B_{1, \varepsilon}+B_{2, \varepsilon}\right)^{2^{\star}-1}-\lambda B_{1, \varepsilon}^{2^{\star}-1}-B_{2, \varepsilon}^{2^{\star}-1}}{\lambda B_{1, \varepsilon}+B_{2, \varepsilon}} .
$$

We assume that $n \geq 6$, and prove the following:

(P1) We fix $x_{1, \varepsilon}=x_{2, \varepsilon}=x_{0}$ in $S^{n}$, we fix $\beta_{1, \varepsilon}=\beta_{1}$, we fix $\lambda=1$, and let $\beta_{2, \varepsilon} \rightarrow 1$. Then the sequence $\left(h_{\varepsilon}\right)$ is bounded in $L^{\infty}\left(S^{n}\right)$, and the sequence $\left(h_{\varepsilon}\right)$ converges to $h_{0}=\Phi_{0}$ in $L^{p}\left(S^{n}\right)$ for all $p \geq 1$.

(P2) We fix $x_{1, \varepsilon}=x_{2, \varepsilon}=x_{0}$ in $S^{n}$, we fix $\beta_{1, \varepsilon}=\beta_{1}$, we fix $\lambda>1$, and let $\beta_{2, \varepsilon} \rightarrow 1$. Then the sequence $\left(h_{\varepsilon}\right)$ is bounded in $L^{\infty}\left(S^{n}\right)$, the sequence $\left(h_{\varepsilon}\right)$ converges to some $h_{0}$ in $L^{p}\left(S^{n}\right)$ for all $p \geq 1$, with $h_{0}>\Phi_{0}$. Moreover, when $n=6$, there exists $\varepsilon_{0}>0$ such that $h_{\varepsilon} \geq \Phi_{0}+\varepsilon_{0}$ for all $\varepsilon$.

(P3) We fix $x_{1, \varepsilon}=x_{0}$ in $S^{n}$, we let $x_{2, \varepsilon}=x_{\varepsilon}$ and $\beta_{1, \varepsilon}=\beta_{2, \varepsilon}=\beta_{\varepsilon}$ such that $\beta_{\varepsilon} \rightarrow 1, d_{h}\left(x_{0}, x_{\varepsilon}\right) \rightarrow 0$ as $\varepsilon \rightarrow 0$ and $d_{h}\left(x_{0}, x_{\varepsilon}\right)>>\left(\beta_{\varepsilon}-1\right)^{1 / 20}$ for all $\varepsilon$. We fix $\lambda=1$. Then the sequence $\left(h_{\varepsilon}\right)$ converges to $h_{0}=\Phi_{0}$ in $C^{1}\left(S^{n}\right)$.

An easy claim is that (P1)-(P3) imply Theorem 0.1. We have indeed, as shown for instance in Druet-Hebey-Robert [7, that

$$
B_{i, \varepsilon}=B_{\varepsilon}^{i}+R_{i, \varepsilon}
$$

where $B_{\varepsilon}^{i}$ is a bubble as in the decomposition (0.1), and $R_{i, \varepsilon} \rightarrow 0$ in $H_{1}^{2}$ as $\varepsilon \rightarrow 0$. If $B_{i, \varepsilon}$ is as above, and $B_{\varepsilon}^{i}$ is as in (0.3), then $x_{\varepsilon}^{i}=x_{i, \varepsilon}$ and

$$
\mu_{\varepsilon}^{i}=\sqrt{\frac{4\left(\beta_{i, \varepsilon}-1\right)}{n(n-2)\left(\beta_{i, \varepsilon}+1\right)}} .
$$

We start with the proof of (P1)-(P2). Then we prove (P3). 
Proof of (P1) and (P2). Let $\left(y_{\varepsilon}\right)$ be a sequence of points in $S^{n}$. It is easily seen that the following propositions hold:

(1) If $B_{2, \varepsilon}\left(y_{\varepsilon}\right) \rightarrow 0$ as $\varepsilon \rightarrow 0$, then

$$
\Phi_{\varepsilon}\left(y_{\varepsilon}\right) \rightarrow\left(\lambda^{2^{\star}-2}-1\right) B_{1}\left(y_{0}\right)^{2^{\star}-2},
$$

where $B_{1}=B_{1, \varepsilon}$ and $y_{0}$ is the limit of the $y_{\varepsilon}$ 's.

(2) If $B_{2, \varepsilon}\left(y_{\varepsilon}\right) \rightarrow C_{0}$ as $\varepsilon \rightarrow 0$, then $y_{\varepsilon} \rightarrow x_{0}$ and

$$
\Phi_{\varepsilon}\left(y_{\varepsilon}\right) \rightarrow \frac{\left(\lambda B_{1}\left(x_{0}\right)+C_{0}\right)^{2^{\star}-1}-\lambda B_{1}\left(x_{0}\right)^{2^{\star}-1}-C_{0}^{2^{\star}-1}}{\lambda B_{1}\left(x_{0}\right)+C_{0}},
$$

where $B_{1}=B_{1, \varepsilon}$.

(3) If $B_{2, \varepsilon}\left(y_{\varepsilon}\right) \rightarrow+\infty$ as $\varepsilon \rightarrow 0$, then $y_{\varepsilon} \rightarrow x_{0}$ and we have that

$$
\Phi_{\varepsilon}\left(y_{\varepsilon}\right) \rightarrow 0 \text { if } n \geq 7 \text { and } \Phi_{\varepsilon}\left(y_{\varepsilon}\right) \rightarrow 2 \lambda B_{1}\left(x_{0}\right) \text { if } n=6,
$$

where $B_{1}=B_{1, \varepsilon}$.

This clearly proves that the $h_{\varepsilon}$ 's are bounded in $L^{\infty}\left(S^{n}\right)$. It is then easily checked that

$$
h_{\varepsilon} \rightarrow h_{0} \text { in } L^{p}\left(S^{n}\right)
$$

for all $p \geq 1$ when $\varepsilon \rightarrow 0$, where

$$
h_{0}=\Phi_{0}+\left(\lambda^{2^{\star}-2}-1\right) B_{1}^{2^{\star}-2}
$$

since $B_{2, \varepsilon} \rightarrow 0$ a.e. Moreover, it is clear that when $n=6$,

$$
\liminf _{\varepsilon \rightarrow 0} \inf _{S^{n}}\left(h_{\varepsilon}-\Phi_{0}\right)>0
$$

when $\lambda>1$. This proves (P1)-(P2).

Now we prove (P3). For the sake of simplicity, we prove that $C^{0}$-convergence holds in all dimensions $n \geq 6$, and restrict ourselves to the case $n=6$ for the $C^{1}$-convergence. Similar arguments to the ones developed below easily give that the $C^{1}$-convergence also holds when $n \geq 7$.

Proof of (P3). We let $\left(y_{\varepsilon}\right)$ be a sequence of points in $S^{n}$. We write that

$$
\Phi_{\varepsilon}\left(y_{\varepsilon}\right)=\frac{\left(B_{1, \varepsilon}\left(y_{\varepsilon}\right)+B_{2, \varepsilon}\left(y_{\varepsilon}\right)\right)^{2^{\star}-1}-B_{1, \varepsilon}\left(y_{\varepsilon}\right)^{2^{\star}-1}-B_{2, \varepsilon}\left(y_{\varepsilon}\right)^{2^{\star}-1}}{B_{1, \varepsilon}\left(y_{\varepsilon}\right)+B_{2, \varepsilon}\left(y_{\varepsilon}\right)} .
$$

We choose $x_{\varepsilon}$ such that

$$
d_{h}\left(x_{\varepsilon}, x_{0}\right)>>\left(\beta_{\varepsilon}-1\right)^{\frac{1}{4}} .
$$

We claim that

$$
\liminf _{\varepsilon \rightarrow 0} B_{2, \varepsilon}\left(y_{\varepsilon}\right)>0 \Longrightarrow \lim _{\varepsilon \rightarrow 0} B_{1, \varepsilon}\left(y_{\varepsilon}\right)=0
$$

and prove the claim in what follows. Let us assume that $B_{1, \varepsilon}\left(y_{\varepsilon}\right) \geq C_{0}$ for some $C_{0}>0$ independent of $\varepsilon$. Then,

$$
\Phi_{0}\left(\beta_{\varepsilon}^{2}-1\right) \geq C_{0}^{\frac{4}{n-2}}\left(\beta_{\varepsilon}-\cos d_{h}\left(x_{0}, y_{\varepsilon}\right)\right)^{2} .
$$

Since $\beta_{\varepsilon} \rightarrow 1$ as $\varepsilon \rightarrow 0$, this clearly implies that $d_{h}\left(x_{0}, y_{\varepsilon}\right) \rightarrow 0$ as $\varepsilon \rightarrow 0$. We then get that

$$
\Phi_{0}\left(\beta_{\varepsilon}^{2}-1\right) \geq C_{0}^{\frac{4}{n-2}}\left(\beta_{\varepsilon}-1+\frac{d_{h}\left(x_{0}, y_{\varepsilon}\right)^{2}}{2}(1+o(1))\right)^{2}
$$


so that

$$
\beta_{\varepsilon}-1+\frac{d_{h}\left(x_{0}, y_{\varepsilon}\right)^{2}}{2}(1+o(1))=O\left(\sqrt{\beta_{\varepsilon}-1}\right) .
$$

This clearly gives that

$$
d_{h}\left(x_{0}, y_{\varepsilon}\right)=O\left(\left(\beta_{\varepsilon}-1\right)^{\frac{1}{4}}\right) .
$$

By (1.1), we then get that

$$
d_{h}\left(x_{\varepsilon}, y_{\varepsilon}\right)=d_{h}\left(x_{0}, x_{\varepsilon}\right)(1+o(1))
$$

so that

$$
\begin{aligned}
B_{2, \varepsilon}\left(y_{\varepsilon}\right)^{\frac{4}{n-2}} & =O\left(\left(\beta_{\varepsilon}-1\right)\left(\beta_{\varepsilon}-\cos \left[d_{h}\left(x_{0}, x_{\varepsilon}\right)(1+o(1))\right]\right)^{-2}\right) \\
& =O\left(\frac{\beta_{\varepsilon}-1}{\left(2\left(\beta_{\varepsilon}-1\right)+d_{h}\left(x_{0}, x_{\varepsilon}\right)^{2}(1+o(1))\right)^{2}}\right) \\
& =o(1)
\end{aligned}
$$

thanks to (1.1), a contradiction. This clearly proves the above claim.

Let us now estimate $\Phi_{\varepsilon}\left(y_{\varepsilon}\right)$. We distinguish two cases.

Case 1. We assume that $B_{2, \varepsilon}\left(y_{\varepsilon}\right)=o\left(B_{1, \varepsilon}\left(y_{\varepsilon}\right)\right)$. Then

$$
\Phi_{\varepsilon}\left(y_{\varepsilon}\right)=\frac{\left(2^{\star}-1\right) B_{1, \varepsilon}\left(y_{\varepsilon}\right)^{2^{\star}-2} B_{2, \varepsilon}\left(y_{\varepsilon}\right)(1+o(1))-B_{2, \varepsilon}\left(y_{\varepsilon}\right)^{2^{\star}-1}}{B_{1, \varepsilon}\left(y_{\varepsilon}\right)(1+o(1))} .
$$

By (1.2), it is clear that $B_{2, \varepsilon}\left(y_{\varepsilon}\right) \rightarrow 0$ as $\varepsilon \rightarrow 0$. If we also have that $B_{1, \varepsilon}\left(y_{\varepsilon}\right) \rightarrow 0$, we can write that

$$
\Phi_{\varepsilon}\left(y_{\varepsilon}\right)=o\left(B_{1, \varepsilon}\left(y_{\varepsilon}\right)^{2^{\star}-2}\right)=o(1) .
$$

If $B_{1, \varepsilon}\left(y_{\varepsilon}\right)$ does not converge to 0 as $\varepsilon \rightarrow 0$, then we get that

$$
\Phi_{\varepsilon}\left(y_{\varepsilon}\right)=o(1)+o\left(B_{1, \varepsilon}\left(y_{\varepsilon}\right)^{2^{\star}-3}\right)=o(1)
$$

since $2^{\star} \leq 3$ when $n \geq 6$. This proves that $\Phi_{\varepsilon}\left(y_{\varepsilon}\right) \rightarrow 0$ as $\varepsilon \rightarrow 0$. By symmetry, it is clear that this conclusion continues to hold when $B_{1, \varepsilon}\left(y_{\varepsilon}\right)=o\left(B_{2, \varepsilon}\left(y_{\varepsilon}\right)\right)$.

Case 2. We assume that $\frac{B_{2, \varepsilon}\left(y_{\varepsilon}\right)}{B_{1, \varepsilon}\left(y_{\varepsilon}\right)} \rightarrow C_{0}$ as $\varepsilon \rightarrow 0$, for some $C_{0}>0$ real. Then

$$
\Phi_{\varepsilon}\left(y_{\varepsilon}\right)=B_{1, \varepsilon}\left(y_{\varepsilon}\right)^{2^{\star}-2}\left(\frac{\left(C_{0}+1\right)^{2^{\star}-1}-C_{0}^{2^{\star}-1}-1}{C_{0}+1}+o(1)\right) .
$$

By $(1.2), B_{2, \varepsilon}\left(y_{\varepsilon}\right) \rightarrow 0$ as $\varepsilon \rightarrow 0$. It follows that $\Phi_{\varepsilon}\left(y_{\varepsilon}\right) \rightarrow 0$ as $\varepsilon \rightarrow 0$.

Thanks to cases 1 and 2 , we get that $\left\|h_{\varepsilon}-\Phi_{0}\right\|_{\infty} \rightarrow 0$ as $\varepsilon \rightarrow 0$. We are now left with the proof that the convergence is $C^{1}$. We restrict ourselves to the case $n=6$. We choose $x_{\varepsilon}$ such that

$$
d_{g}\left(x_{\varepsilon}, x_{0}\right)>>\left(\beta_{\varepsilon}-1\right)^{\frac{n-2}{4(n-1)}}
$$

and let $\left(y_{\varepsilon}\right)$ be a sequence of points in $S^{n}$. We claim that

$$
\liminf _{\varepsilon \rightarrow 0}\left|\nabla B_{2, \varepsilon}\left(y_{\varepsilon}\right)\right|>0 \Longrightarrow \lim _{\varepsilon \rightarrow 0} \frac{B_{2, \varepsilon}\left(y_{\varepsilon}\right)}{B_{1, \varepsilon}\left(y_{\varepsilon}\right)}=+\infty
$$


and

$$
\begin{aligned}
& \liminf _{\varepsilon \rightarrow 0}\left|\nabla B_{2, \varepsilon}\left(y_{\varepsilon}\right)\right|>0 \\
& \quad \Longrightarrow B_{1, \varepsilon}\left(y_{\varepsilon}\right)=(n(n-2))^{\frac{n-2}{4}}\left(\beta_{\varepsilon}^{2}-1\right)^{\frac{n-2}{4}} d_{h}\left(y_{\varepsilon}, x_{0}\right)^{2-n}(1+o(1)) .
\end{aligned}
$$

We prove these claims. We assume that $\left|\nabla B_{2, \varepsilon}\left(y_{\varepsilon}\right)\right| \geq C_{0}$ for some $C_{0}>0$ independent of $\varepsilon$. Then

$$
\left(\beta_{\varepsilon}-\cos d_{h}\left(x_{\varepsilon}, y_{\varepsilon}\right)\right)^{\frac{n}{2}}=O\left(\left(\beta_{\varepsilon}-1\right)^{\frac{n-2}{4}} \sin d_{h}\left(x_{\varepsilon}, y_{\varepsilon}\right)\right) .
$$

This implies that $d_{h}\left(x_{\varepsilon}, y_{\varepsilon}\right) \rightarrow 0$ as $\varepsilon \rightarrow 0$ and thus that

$$
\beta_{\varepsilon}-1+\frac{d_{h}\left(x_{\varepsilon}, y_{\varepsilon}\right)^{2}}{2}(1+o(1))=O\left(\left(\beta_{\varepsilon}-1\right)^{\frac{n-2}{2 n}} d_{h}\left(x_{\varepsilon}, y_{\varepsilon}\right)^{\frac{2}{n}}\right) \text {. }
$$

This clearly leads to

$$
d_{h}\left(x_{\varepsilon}, y_{\varepsilon}\right)=O\left(\left(\beta_{\varepsilon}-1\right)^{\frac{n-2}{4(n-1)}}\right) .
$$

By (1.3), we thus get that

$$
d_{h}\left(x_{0}, y_{\varepsilon}\right)=d_{h}\left(x_{0}, x_{\varepsilon}\right)(1+o(1))
$$

which obviously proves (1.5). Thanks to (1.6) we can then write that

$$
\begin{aligned}
\frac{B_{2, \varepsilon}\left(y_{\varepsilon}\right)}{B_{1, \varepsilon}\left(y_{\varepsilon}\right)} \geq & 4^{-\frac{n-2}{4}}\left(\beta_{\varepsilon}-\cos d_{h}\left(x_{\varepsilon}, y_{\varepsilon}\right)\right)^{1-\frac{n}{2}} d_{h}\left(x_{0}, x_{\varepsilon}\right)^{n-2}(1+o(1)) \\
\geq & 4^{-\frac{n-2}{4}}\left(\beta_{\varepsilon}-1+\frac{d_{h}\left(x_{\varepsilon}, y_{\varepsilon}\right)^{2}}{2}(1+o(1))\right)^{1-\frac{n}{2}} \\
& \quad \times d_{h}\left(x_{0}, x_{\varepsilon}\right)^{n-2}(1+o(1)) \\
\geq & C\left(\beta_{\varepsilon}-1\right)^{-\frac{(n-2)^{2}}{4(n-1)}} d_{h}\left(x_{0}, x_{\varepsilon}\right)^{n-2}(1+o(1)),
\end{aligned}
$$

where $C>0$ is independent of $\varepsilon$. This proves (1.4) thanks to (1.3). We assume now that $n=6$. Then

$$
\nabla h_{\varepsilon}\left(y_{\varepsilon}\right)=2 \frac{B_{2, \varepsilon}\left(y_{\varepsilon}\right)^{2} \nabla B_{1, \varepsilon}\left(y_{\varepsilon}\right)+B_{1, \varepsilon}\left(y_{\varepsilon}\right)^{2} \nabla B_{2, \varepsilon}\left(y_{\varepsilon}\right)}{\left(B_{1, \varepsilon}\left(y_{\varepsilon}\right)+B_{2, \varepsilon}\left(y_{\varepsilon}\right)\right)^{2}} .
$$

We distinguish two cases.

Case 1. We assume that $\frac{B_{2, \varepsilon}\left(y_{\varepsilon}\right)}{B_{1, \varepsilon}\left(y_{\varepsilon}\right)} \rightarrow 0$ as $\varepsilon \rightarrow 0$. Then

$$
\left|\nabla h_{\varepsilon}\left(y_{\varepsilon}\right)\right|=O\left(\frac{B_{2, \varepsilon}\left(y_{\varepsilon}\right)^{2}}{B_{1, \varepsilon}\left(y_{\varepsilon}\right)^{2}}\left|\nabla B_{1, \varepsilon}\left(y_{\varepsilon}\right)\right|\right)+O\left(\left|\nabla B_{2, \varepsilon}\left(y_{\varepsilon}\right)\right|\right) .
$$

By (1.4), we have that $\left|\nabla B_{2, \varepsilon}\left(y_{\varepsilon}\right)\right| \rightarrow 0$ as $\varepsilon \rightarrow 0$. As a starting point, we assume that $\left|\nabla B_{1, \varepsilon}\left(y_{\varepsilon}\right)\right|=O(1)$. Then we get that

$$
\left|\nabla h_{\varepsilon}\left(y_{\varepsilon}\right)\right|=o(1) \text {. }
$$

Assume now that $\left|\nabla B_{1, \varepsilon}\left(y_{\varepsilon}\right)\right| \rightarrow+\infty$ as $\varepsilon \rightarrow 0$. Then, thanks to the analogue of (1.5) for $B_{1, \varepsilon}$, noting that our assumption implies (see above) that

$$
d_{h}\left(x_{\varepsilon}, y_{\varepsilon}\right)=d_{h}\left(x_{0}, x_{\varepsilon}\right)(1+o(1))
$$

we get that

$$
B_{2, \varepsilon}\left(y_{\varepsilon}\right)=O\left(\left(\beta_{\varepsilon}-1\right) d_{h}\left(x_{\varepsilon}, x_{0}\right)^{-4}\right)
$$


Since $\left|\nabla B_{1, \varepsilon}\left(y_{\varepsilon}\right)\right| \rightarrow+\infty$ as $\varepsilon \rightarrow 0$, we have that $d_{h}\left(x_{0}, y_{\varepsilon}\right) \rightarrow 0$ as $\varepsilon \rightarrow 0$ so that

$$
\begin{aligned}
\frac{\left|\nabla B_{1, \varepsilon}\left(y_{\varepsilon}\right)\right|}{B_{1, \varepsilon}\left(y_{\varepsilon}\right)^{2}} & =O\left(\frac{d_{h}\left(x_{0}, y_{\varepsilon}\right)}{\beta_{\varepsilon}-1}\left(\beta_{\varepsilon}-1+\frac{d_{h}\left(x_{0}, y_{\varepsilon}\right)^{2}}{2}(1+o(1))\right)\right) \\
& =O\left(d_{h}\left(x_{0}, y_{\varepsilon}\right)\right)+O\left(\frac{d_{h}\left(x_{0}, y_{\varepsilon}\right)^{3}}{\beta_{\varepsilon}-1}\right) .
\end{aligned}
$$

As in the proof of (1.4) and (1.5), we can prove that the analog of (1.6) for $B_{1, \varepsilon}$ holds. Namely we have that

$$
d_{h}\left(x_{0}, y_{\varepsilon}\right)=O\left(\left(\beta_{\varepsilon}-1\right)^{\frac{1}{5}}\right) .
$$

Thus we get that

$$
\frac{\left|\nabla B_{1, \varepsilon}\left(y_{\varepsilon}\right)\right|}{B_{1, \varepsilon}\left(y_{\varepsilon}\right)^{2}}=O\left(\left(\beta_{\varepsilon}-1\right)^{-\frac{2}{5}}\right) .
$$

Thanks to (1.7), we then get that

$$
B_{2, \varepsilon}\left(y_{\varepsilon}\right) \frac{\left|\nabla B_{1, \varepsilon}\left(y_{\varepsilon}\right)\right|}{B_{1, \varepsilon}\left(y_{\varepsilon}\right)^{2}}=O\left(\left(\beta_{\varepsilon}-1\right)^{\frac{3}{5}} d_{h}\left(x_{\varepsilon}, x_{0}\right)^{-4}\right) .
$$

Choosing $x_{\varepsilon}$ such that

$$
d_{h}\left(x_{\varepsilon}, x_{0}\right)>>\left(\beta_{\varepsilon}-1\right)^{\frac{3}{20}}
$$

we get that $\left|\nabla h_{\varepsilon}\left(y_{\varepsilon}\right)\right| \rightarrow 0$ as $\varepsilon \rightarrow 0$. By symmetry, the conclusion holds also if we assume that $\frac{B_{1, \varepsilon}\left(y_{\varepsilon}\right)}{B_{2, \varepsilon}\left(y_{\varepsilon}\right)} \rightarrow 0$ as $\varepsilon \rightarrow 0$.

Case 2. We assume that $\frac{B_{2, \varepsilon}\left(y_{\varepsilon}\right)}{B_{1, \varepsilon}\left(y_{\varepsilon}\right)} \rightarrow C_{0}$ as $\varepsilon \rightarrow 0$ for some $C_{0}>0$ real. Then

$$
\left|\nabla h_{\varepsilon}\left(y_{\varepsilon}\right)\right|=O\left(\left|\nabla B_{1, \varepsilon}\left(y_{\varepsilon}\right)\right|\right)+O\left(\left|\nabla B_{2, \varepsilon}\left(y_{\varepsilon}\right)\right|\right) \text {. }
$$

Thanks to (1.4), and its analogue for $B_{1, \varepsilon}$, it is easily checked that $\left|\nabla B_{1, \varepsilon}\left(y_{\varepsilon}\right)\right| \rightarrow 0$ and $\left|\nabla B_{2, \varepsilon}\left(y_{\varepsilon}\right)\right| \rightarrow 0$ as $\varepsilon \rightarrow 0$. Thus $\left|\nabla h_{\varepsilon}\left(y_{\varepsilon}\right)\right| \rightarrow 0$ as $\varepsilon \rightarrow 0$.

Thanks to these two cases, the convergence of the $h_{\varepsilon}$ 's to $\Phi_{0}$ is $C^{1}$ when $n=6$. Similar arguments give that we also have that $h_{\varepsilon} \rightarrow \Phi_{0}$ in $C^{1}\left(S^{n}\right)$ when $n \geq 7$. This ends the proof of (P3).

The bubbles in (P3) interact in a weak sense at the $C^{0}$-level. We define the strong interaction at the $C^{0}$-level of two bubbles $\left(B_{\varepsilon}\right)$ and $\left(\tilde{B}_{\varepsilon}\right)$ by the existence of $C>0$ and a sequence $\left(\hat{x}_{\varepsilon}\right)$ of points in $M$ such that

$$
\liminf _{\varepsilon \rightarrow 0} B_{\varepsilon}\left(\hat{x}_{\varepsilon}\right) \geq C \text { and } \liminf _{\varepsilon \rightarrow 0} \tilde{B}_{\varepsilon}\left(\hat{x}_{\varepsilon}\right) \geq C .
$$

An easy claim is that there exist examples of $h_{\varepsilon}$ 's and $u_{\varepsilon}$ 's satisfying (0.5) such that the sequence $\left(h_{\varepsilon}\right)$ is bounded in $L^{\infty}\left(S^{n}\right)$, the sequence $\left(h_{\varepsilon}\right)$ converges to $h_{0}=\Phi_{0}$ in $L^{p}\left(S^{n}\right)$ for all $p \geq 1$, and the sequence $u_{\varepsilon}=B_{1, \varepsilon}+B_{2, \varepsilon}$ is such that $\left(B_{1, \varepsilon}\right)$ and $\left(B_{2, \varepsilon}\right)$ interact strongly at the $C^{0}$-level. For the sake of simplicity, we prove this claim when $n=6$. We let $\beta_{1, \varepsilon}=\beta_{2, \varepsilon}=\beta_{\varepsilon}$, where $\beta_{\varepsilon}>1$ is such that $\beta_{\varepsilon} \rightarrow 1$ as $\varepsilon \rightarrow 0$, and let $\left(x_{1, \varepsilon}\right)$ and $\left(x_{2, \varepsilon}\right)$ be such that $d_{h}\left(x_{1, \varepsilon}, x_{2, \varepsilon}\right)=2\left(\beta_{\varepsilon}-1\right)^{1 / 4}$ for all $\varepsilon$. As above, we let $B_{i, \varepsilon}, i=1,2$, be such that

$$
B_{i, \varepsilon}(x)=6\left(\beta_{\varepsilon}^{2}-1\right)\left(\beta_{\varepsilon}-\cos d_{h}\left(x_{i, \varepsilon}, x\right)\right)^{-2}
$$

and set $u_{\varepsilon}=B_{1, \varepsilon}+B_{2, \varepsilon}$. Then,

$$
\Delta_{h} u_{\varepsilon}+h_{\varepsilon} u_{\varepsilon}=u_{\varepsilon}^{2^{\star}-1}
$$


for all $\varepsilon$, where $h_{\varepsilon}=\Phi_{0}+\Phi_{\varepsilon}$ and $\Phi_{\varepsilon}=2 B_{1, \varepsilon} B_{2, \varepsilon} /\left(B_{1, \varepsilon}+B_{2, \varepsilon}\right)$. Let $\delta>0$ be such that $\cos \theta \leq 1-\frac{\theta^{2}}{4}$ if $|\theta| \leq \delta$. It is easily checked that $\Phi_{\varepsilon} \rightarrow 0$ in $C^{0}\left(S^{n} \backslash X_{\varepsilon}\right)$ as $\varepsilon \rightarrow 0$, where $X_{\varepsilon}=B_{x_{1, \varepsilon}}(\delta) \cap B_{x_{2, \varepsilon}}(\delta)$. Noting that

$$
\begin{aligned}
\beta_{\varepsilon}-\cos d_{h}\left(x_{i, \varepsilon}, x\right) & \geq \beta_{\varepsilon}-1+\frac{1}{4} \sqrt{\beta_{\varepsilon}-1} \\
& \geq \frac{1}{8} \sqrt{\beta_{\varepsilon}-1}
\end{aligned}
$$

if $\left(\beta_{\varepsilon}-1\right)^{1 / 4} \leq d_{h}\left(x_{i, \varepsilon}, x\right) \leq \delta$, we also have that there exists $C>0$ such that $\Phi_{\varepsilon}(x) \leq C$ for all $x \in \bigcup_{i=1,2} B_{x_{i, \varepsilon}}(\delta) \backslash B_{x_{i, \varepsilon}}\left(\left(\beta_{\varepsilon}-1\right)^{1 / 4}\right)$. We have that $d_{h}\left(x_{1, \varepsilon}, x_{2, \varepsilon}\right)=2\left(\beta_{\varepsilon}-1\right)^{1 / 4}$. Hence,

$$
B_{x_{1, \varepsilon}}\left(\left(\beta_{\varepsilon}-1\right)^{1 / 4}\right) \cap B_{x_{2, \varepsilon}}\left(\left(\beta_{\varepsilon}-1\right)^{1 / 4}\right)=\emptyset .
$$

This proves that the $\Phi_{\varepsilon}$ 's are bounded in $L^{\infty}\left(S^{n}\right)$. Let $\delta_{\varepsilon}>0, \delta_{\varepsilon} \rightarrow 0$ as $\varepsilon \rightarrow 0$, be such that $\left(\beta_{\varepsilon}-1\right)^{1 / 8} \leq \delta_{\varepsilon} \leq \delta$. Then,

$$
\beta_{\varepsilon}-\cos d_{h}\left(x_{i, \varepsilon}, x\right) \geq \frac{1}{8}\left(\beta_{\varepsilon}-1\right)^{1 / 4}
$$

if $d_{h}\left(x_{i, \varepsilon}, x\right) \geq \delta_{\varepsilon}$, so that $\Phi_{\varepsilon} \rightarrow 0$ in $C^{0}\left(S^{n} \backslash \hat{X}_{\varepsilon}\right)$ as $\varepsilon \rightarrow 0$, where $\hat{X}_{\varepsilon}$ is given by $\hat{X}_{\varepsilon}=B_{x_{1, \varepsilon}}\left(\delta_{\varepsilon}\right) \cup B_{x_{2, \varepsilon}}\left(\delta_{\varepsilon}\right)$. In particular, since $\left(\Phi_{\varepsilon}\right)$ is bounded in $L^{\infty}\left(S^{n}\right)$, we get that for any $p \geq 1, \Phi_{\varepsilon} \rightarrow 0$ in $L^{p}\left(S^{n}\right)$ as $\varepsilon \rightarrow 0$. It follows that the above $h_{\varepsilon}$ 's are bounded in $L^{\infty}\left(S^{n}\right)$ and that they converge to $h_{0}=\Phi_{0}$ in $L^{p}\left(S^{n}\right)$ for all $p \geq 1$. Now we let $x_{\varepsilon}$ be such that

$$
d_{h}\left(x_{1, \varepsilon}, x_{\varepsilon}\right)=d_{h}\left(x_{2, \varepsilon}, x_{\varepsilon}\right)=\left(\beta_{\varepsilon}-1\right)^{1 / 4} .
$$

Then $B_{1, \varepsilon}\left(x_{\varepsilon}\right)=B_{2, \varepsilon}\left(x_{\varepsilon}\right)$, and we easily get that there exists $C>0$ such that for $\varepsilon>0$ sufficiently small,

$$
\frac{\beta_{\varepsilon}^{2}-1}{\left(\beta_{\varepsilon}-\cos d_{h}\left(x_{i, \varepsilon}, x_{\varepsilon}\right)\right)^{2}} \geq C
$$

for all $i=1,2$. This proves that $\left(B_{1, \varepsilon}\right)$ and $\left(B_{2, \varepsilon}\right)$ interact strongly at the $C^{0}$-level, and the above claim. Then it is easily checked that the representatives $B_{\varepsilon}^{i}$ of the $B_{i, \varepsilon}$ 's also interact strongly at the $C^{0}$-level.

As a remark, several of the preceding results can be extended to more than one or two bubbles. For instance, if we assume $n=6$, and let $u_{\varepsilon}=\sum B_{i, \varepsilon}$ where the $B_{i, \varepsilon}$ 's are as above, then the $u_{\varepsilon}$ 's satisfy $(0.5)$ with

$$
h_{\varepsilon}=\Phi_{0}+2 \frac{\sum_{i<j} B_{i, \varepsilon} B_{j, \varepsilon}}{\sum_{i} B_{i, \varepsilon}}
$$

where $\Phi_{0} \equiv \frac{n(n-2)}{4}$ is as above. When $n \neq 6$, and $u_{\varepsilon}=\sum B_{i, \varepsilon}$, we have that $h_{\varepsilon}=\Phi_{0}+\Phi_{\varepsilon}$, where

$$
\Phi_{\varepsilon}=\frac{\left(\sum B_{i, \varepsilon}\right)^{2^{\star}-1}-\sum B_{i, \varepsilon}^{2^{\star}-1}}{\sum B_{i, \varepsilon}}
$$

and $\Phi_{0} \equiv \frac{n(n-2)}{4}$ is as above. 


\section{Proof of Theorem 0.2}

We prove Theorem 0.2 in what follows. Here again, for the sake of simplicity, we prove that $C^{0}$-convergence holds in all dimensions $n \geq 6$, and restrict ourselves to the case $n=6$ for the $C^{1}$-convergence. Similar arguments to the ones developed below easily give the result when $n \geq 7$. We assume $n \geq 6$. We let $\left(N_{\varepsilon}\right)$ be a sequence of integers such that $N_{\varepsilon} \rightarrow+\infty$ as $\varepsilon \rightarrow 0$. Given $\varepsilon>0$, we also let $x_{1, \varepsilon}, \ldots, x_{N_{\varepsilon}, \varepsilon}$ be $N_{\varepsilon}$ points in $M$ such that $x_{i, \varepsilon} \neq x_{j, \varepsilon}$ when $i \neq j$. We define

$$
d_{\varepsilon}=\min _{i \neq j} d_{h}\left(x_{i, \varepsilon}, x_{j, \varepsilon}\right) .
$$

Then we set

$$
u_{\varepsilon}=\sum_{i=1}^{N_{\varepsilon}} B_{i, \varepsilon}
$$

where the $B_{i, \varepsilon}$ 's are as in section 1 , the $x_{i, \varepsilon}$ 's are as above, the $\beta_{i, \varepsilon}$ 's are such that $\beta_{i, \varepsilon}=\beta_{\varepsilon}$ for all $i$, and the $\beta_{\varepsilon}$ 's are such that

$$
\begin{aligned}
& \left(\beta_{\varepsilon}-1\right) N_{\varepsilon}^{9} \rightarrow 0 \text { as } \varepsilon \rightarrow 0, \text { and } \\
& \beta_{\varepsilon}-1 \leq \frac{d_{\varepsilon}^{8}}{N_{\varepsilon}}
\end{aligned}
$$

It is easily checked that $u_{\varepsilon} \in C^{\infty}\left(S^{n}\right)$, that $u_{\varepsilon}>0$, and that

$$
\Delta_{h} u_{\varepsilon}+h_{\varepsilon} u_{\varepsilon}=u_{\varepsilon}^{2^{\star}-1}
$$

in $S^{n}$, where $h_{\varepsilon}=\Phi_{0}+\Phi_{\varepsilon}$ and

$$
\Phi_{\varepsilon}=\frac{\left(\sum_{i=1}^{N_{\varepsilon}} B_{i, \varepsilon}\right)^{2^{\star}-1}-\sum_{i=1}^{N_{\varepsilon}} B_{i, \varepsilon}^{2^{\star}-1}}{\sum_{i=1}^{N_{\varepsilon}} B_{i, \varepsilon}} .
$$

In particular, the $u_{\varepsilon}$ 's and $h_{\varepsilon}$ 's satisfy $(0.5)$. First we prove that $\Phi_{\varepsilon} \rightarrow 0$ in $C^{0}\left(S^{n}\right)$ as $\varepsilon \rightarrow 0$, and thus that $h_{\varepsilon} \rightarrow \Phi_{0}$ in $C^{0}\left(S^{n}\right)$ as $\varepsilon \rightarrow 0$. Since $2^{\star}-1 \geq 1$, it is clear that $\Phi_{\varepsilon} \geq 0$ in $S^{n}$. We let $x_{\varepsilon} \in S^{n}$ be such that

$$
\Phi_{\varepsilon}\left(x_{\varepsilon}\right)=\max _{S^{n}} \Phi_{\varepsilon}
$$

Up to renumbering, we may assume that

$$
B_{1, \varepsilon}\left(x_{\varepsilon}\right) \geq B_{i, \varepsilon}\left(x_{\varepsilon}\right)
$$

for all $i=2, \ldots, N_{\varepsilon}$. For $t \geq 0$, and $p=2^{\star}-1,(1+t)^{p} \leq 1+p t+t^{p}$ since $2 \leq 2^{\star} \leq 3$ when $n \geq 6$. Writing that

$$
\begin{aligned}
& B_{1, \varepsilon}\left(x_{\varepsilon}\right)^{2^{\star}-2} \sum_{i=2}^{N_{\varepsilon}} B_{i, \varepsilon}\left(x_{\varepsilon}\right) \\
& \quad=B_{1, \varepsilon}\left(x_{\varepsilon}\right) \sum_{i=2}^{N_{\varepsilon}} B_{i, \varepsilon}\left(x_{\varepsilon}\right)^{2^{\star}-2}\left(B_{i, \varepsilon}\left(x_{\varepsilon}\right) B_{1, \varepsilon}\left(x_{\varepsilon}\right)^{-1}\right)^{3-2^{\star}} \\
& \quad \leq B_{1, \varepsilon}\left(x_{\varepsilon}\right) \sum_{i=2}^{N_{\varepsilon}} B_{i, \varepsilon}\left(x_{\varepsilon}\right)^{2^{\star}-2}
\end{aligned}
$$


we then get that

$$
\begin{aligned}
& \left(\sum_{i=1}^{N_{\varepsilon}} B_{i, \varepsilon}\left(x_{\varepsilon}\right)\right) \Phi_{\varepsilon}\left(x_{\varepsilon}\right) \\
& \quad \leq\left(\sum_{i=2}^{N_{\varepsilon}} B_{i, \varepsilon}\left(x_{\varepsilon}\right)\right)^{2^{\star}-1}+\left(2^{\star}-1\right) B_{1, \varepsilon}\left(x_{\varepsilon}\right)^{2^{\star}-2} \sum_{i=2}^{N_{\varepsilon}} B_{i, \varepsilon}\left(x_{\varepsilon}\right) \\
& \quad \leq\left(\sum_{i=2}^{N_{\varepsilon}} B_{i, \varepsilon}\left(x_{\varepsilon}\right)\right)\left(\sum_{i=2}^{N_{\varepsilon}} B_{i, \varepsilon}\left(x_{\varepsilon}\right)\right)^{2^{\star}-2}+\left(2^{\star}-1\right) B_{1, \varepsilon}\left(x_{\varepsilon}\right) \sum_{i=2}^{N_{\varepsilon}} B_{i, \varepsilon}\left(x_{\varepsilon}\right)^{2^{\star}-2} \\
& \quad \leq\left(\sum_{i=2}^{N_{\varepsilon}} B_{i, \varepsilon}\left(x_{\varepsilon}\right)\right)\left(\left(\sum_{i=2}^{N_{\varepsilon}} B_{i, \varepsilon}\left(x_{\varepsilon}\right)\right)^{2^{\star}-2}+\left(2^{\star}-1\right) \sum_{i=2}^{N_{\varepsilon}} B_{i, \varepsilon}\left(x_{\varepsilon}\right)^{2^{\star}-2}\right)
\end{aligned}
$$

and since $2^{\star}-2 \leq 1$ when $n \geq 6$, we get in particular that

$$
\Phi_{\varepsilon}\left(x_{\varepsilon}\right) \leq 2^{\star} \sum_{i=2}^{N_{\varepsilon}} B_{i, \varepsilon}\left(x_{\varepsilon}\right)^{2^{\star}-2} .
$$

If $N_{\varepsilon} B_{1, \varepsilon}\left(x_{\varepsilon}\right)^{2^{\star}-2} \rightarrow 0$ as $\varepsilon \rightarrow 0$, since $B_{1, \varepsilon}\left(x_{\varepsilon}\right) \geq B_{i, \varepsilon}\left(x_{\varepsilon}\right)$ for all $i=2, \ldots, N_{\varepsilon}$, we easily obtain that $\Phi_{\varepsilon}\left(x_{\varepsilon}\right) \rightarrow 0$ as $\varepsilon \rightarrow 0$. Let us now assume that

$$
N_{\varepsilon} B_{1, \varepsilon}\left(x_{\varepsilon}\right)^{2^{\star}-2} \geq \delta_{0}
$$

for some $\delta_{0}>0$. Then,

$$
N_{\varepsilon} \frac{n(n-2)}{4}\left(\beta_{\varepsilon}^{2}-1\right)\left(\beta_{\varepsilon}-\cos d_{h}\left(x_{1, \varepsilon}, x_{\varepsilon}\right)\right)^{-2} \geq \delta_{0}
$$

and since $N_{\varepsilon}\left(\beta_{\varepsilon}-1\right) \rightarrow 0$ (see $\left.(2.2)\right)$ we can write that

$$
d_{h}\left(x_{1, \varepsilon}, x_{\varepsilon}\right) \leq\left(N_{\varepsilon}\left(\beta_{\varepsilon}-1\right)\right)^{\frac{1}{5}}
$$

for $\varepsilon>0$ small. By $(2.2), d_{\varepsilon} \geq\left(N_{\varepsilon}\left(\beta_{\varepsilon}-1\right)\right)^{\frac{1}{8}}$. It follows (see the definition (2.1) of $\left.d_{\varepsilon}\right)$ that

$$
d_{h}\left(x_{i, \varepsilon}, x_{\varepsilon}\right) \geq\left(N_{\varepsilon}\left(\beta_{\varepsilon}-1\right)\right)^{\frac{1}{6}}
$$

for all $i=2, \ldots, N_{\varepsilon}$, and $\varepsilon>0$ small. In particular,

$$
\sum_{i=2}^{N_{\varepsilon}} B_{i, \varepsilon}\left(x_{\varepsilon}\right)^{2^{\star}-2} \leq \frac{N_{\varepsilon} n(n-2)\left(\beta_{\varepsilon}^{2}-1\right)}{4\left(\beta_{\varepsilon}-\cos \left(\left(N_{\varepsilon}\left(\beta_{\varepsilon}-1\right)\right)^{\frac{1}{6}}\right)\right)^{2}}
$$

and we get that

$$
\sum_{i=2}^{N_{\varepsilon}} B_{i, \varepsilon}\left(x_{\varepsilon}\right)^{2^{\star}-2}=O\left(\left(N_{\varepsilon}\left(\beta_{\varepsilon}-1\right)\right)^{\frac{1}{3}}\right)=o(1) .
$$

By (2.3) and (2.4) we then obtain that $\Phi_{\varepsilon}\left(x_{\varepsilon}\right) \rightarrow 0$ as $\varepsilon \rightarrow 0$. This proves that $\Phi_{\varepsilon} \rightarrow 0$ in $C^{0}\left(S^{n}\right)$ as $\varepsilon \rightarrow 0$, and thus that

$$
h_{\varepsilon} \rightarrow \Phi_{0} \text { in } C^{0}\left(S^{n}\right)
$$


as $\varepsilon \rightarrow 0$. Now we assume that $n=6$, and prove that $\Phi_{\varepsilon} \rightarrow 0$ in $C^{1}\left(S^{6}\right)$ as $\varepsilon \rightarrow 0$, so that $h_{\varepsilon} \rightarrow \Phi_{0}$ in $C^{1}\left(S^{6}\right)$ as $\varepsilon \rightarrow 0$. We write that

$$
\nabla \Phi_{\varepsilon}=\sum_{j=1}^{N_{\varepsilon}} \frac{\left(\sum_{i \neq j} B_{i, \varepsilon}^{2}+\left(\sum_{i \neq j} B_{i, \varepsilon}\right)^{2}\right) \nabla B_{j, \varepsilon}}{\left(\sum_{i=1}^{N_{\varepsilon}} B_{i, \varepsilon}\right)^{2}}
$$

and then that

$$
\left|\nabla \Phi_{\varepsilon}\right| \leq 2 \sum_{j=1}^{N_{\varepsilon}} \frac{\left(\sum_{i \neq j} B_{i, \varepsilon}\right)^{2}\left|\nabla B_{j, \varepsilon}\right|}{\left(\sum_{i=1}^{N_{\varepsilon}} B_{i, \varepsilon}\right)^{2}} .
$$

We let $x_{\varepsilon} \in S^{6}$ be such that

$$
\left|\nabla \Phi_{\varepsilon}\left(x_{\varepsilon}\right)\right|=\max _{S^{6}}\left|\nabla \Phi_{\varepsilon}\right| .
$$

Up to renumbering, we may assume that

$$
\frac{\left(\sum_{i \neq j} B_{i, \varepsilon}\left(x_{\varepsilon}\right)\right)^{2}\left|\nabla B_{j, \varepsilon}\left(x_{\varepsilon}\right)\right|}{\left(\sum_{i=1}^{N_{\varepsilon}} B_{i, \varepsilon}\left(x_{\varepsilon}\right)\right)^{2}} \leq \frac{\left(\sum_{i=2}^{N_{\varepsilon}} B_{i, \varepsilon}\left(x_{\varepsilon}\right)\right)^{2}\left|\nabla B_{1, \varepsilon}\left(x_{\varepsilon}\right)\right|}{\left(\sum_{i=1}^{N_{\varepsilon}} B_{i, \varepsilon}\left(x_{\varepsilon}\right)\right)^{2}}
$$

for all $\varepsilon>0$ and all $j=2, \ldots, N_{\varepsilon}$. Then,

$$
\left\|\nabla \Phi_{\varepsilon}\right\|_{L^{\infty}\left(S^{6}\right)} \leq N_{\varepsilon}\left|\nabla B_{1, \varepsilon}\left(x_{\varepsilon}\right)\right| \frac{\left(\sum_{i=2}^{N_{\varepsilon}} B_{i, \varepsilon}\left(x_{\varepsilon}\right)\right)^{2}}{\left(\sum_{i=1}^{N_{\varepsilon}} B_{i, \varepsilon}\left(x_{\varepsilon}\right)\right)^{2}} .
$$

If $N_{\varepsilon}\left|\nabla B_{1, \varepsilon}\left(x_{\varepsilon}\right)\right| \rightarrow 0$ as $\varepsilon \rightarrow 0$, we clearly get that $\left\|\nabla \Phi_{\varepsilon}\right\|_{L^{\infty}\left(S^{6}\right)} \rightarrow 0$ as $\varepsilon \rightarrow 0$.

Let us now assume that

for some $\delta_{0}>0$. Then

$$
N_{\varepsilon}\left|\nabla B_{1, \varepsilon}\left(x_{\varepsilon}\right)\right| \geq \delta_{0}
$$

$$
12 N_{\varepsilon}\left(\beta_{\varepsilon}^{2}-1\right) \frac{\sin d_{h}\left(x_{1, \varepsilon}, x_{\varepsilon}\right)}{\left(\beta_{\varepsilon}-\cos d_{h}\left(x_{1, \varepsilon}, x_{\varepsilon}\right)\right)^{3}} \geq \delta_{0}
$$

and since $N_{\varepsilon}\left(\beta_{\varepsilon}-1\right) \rightarrow 0$ (see $(2.2)$ ) we can write that $d_{h}\left(x_{1, \varepsilon}, x_{\varepsilon}\right) \rightarrow 0$ and then that

$$
d_{h}\left(x_{1, \varepsilon}, x_{\varepsilon}\right)=O\left(\left(N_{\varepsilon}\left(\beta_{\varepsilon}-1\right)\right)^{\frac{1}{5}}\right) .
$$

By $(2.2), d_{\varepsilon} \geq\left(N_{\varepsilon}\left(\beta_{\varepsilon}-1\right)\right)^{\frac{1}{8}}$. It follows (see the definition (2.1) of $d_{\varepsilon}$ ) that

$$
d_{h}\left(x_{i, \varepsilon}, x_{\varepsilon}\right) \geq\left(N_{\varepsilon}\left(\beta_{\varepsilon}-1\right)\right)^{\frac{1}{6}}
$$

for all $i=2, \ldots, N_{\varepsilon}$, and $\varepsilon>0$ small. Hence,

$$
\left(\sum_{i=2}^{N_{\varepsilon}} B_{i, \varepsilon}\left(x_{\varepsilon}\right)\right)^{2}=O\left(N_{\varepsilon}^{2}\left(\beta_{\varepsilon}-1\right)^{2}\left(\beta_{\varepsilon}-\cos \left(\left(N_{\varepsilon}\left(\beta_{\varepsilon}-1\right)\right)^{\frac{1}{6}}\right)\right)^{-4}\right)
$$

and we get that

$$
\left(\sum_{i=2}^{N_{\varepsilon}} B_{i, \varepsilon}\left(x_{\varepsilon}\right)\right)^{2}=O\left(N_{\varepsilon}^{\frac{2}{3}}\left(\beta_{\varepsilon}-1\right)^{\frac{2}{3}}\right)
$$


Independently,

$$
\begin{aligned}
\frac{\left|\nabla B_{1, \varepsilon}\left(x_{\varepsilon}\right)\right|}{B_{1, \varepsilon}\left(x_{\varepsilon}\right)^{2}} & =O\left(\frac{d_{h}\left(x_{1, \varepsilon}, x_{\varepsilon}\right)}{\beta_{\varepsilon}-1}\left(\beta_{\varepsilon}-\cos d_{h}\left(x_{1, \varepsilon}, x_{\varepsilon}\right)\right)\right) \\
& =O\left(d_{h}\left(x_{1, \varepsilon}, x_{\varepsilon}\right)\right)+O\left(\frac{d_{h}\left(x_{1, \varepsilon}, x_{\varepsilon}\right)^{3}}{\beta_{\varepsilon}-1}\right) .
\end{aligned}
$$

Thanks to (2.6), this implies that

$$
\frac{\left|\nabla B_{1, \varepsilon}\left(x_{\varepsilon}\right)\right|}{B_{1, \varepsilon}\left(x_{\varepsilon}\right)^{2}}=O\left(N_{\varepsilon}^{\frac{3}{5}}\left(\beta_{\varepsilon}-1\right)^{-\frac{2}{5}}\right) .
$$

Coming back to (2.5), thanks to (2.7) and (2.8), we then get that

$$
\left\|\nabla \Phi_{\varepsilon}\right\|_{L^{\infty}\left(S^{6}\right)}=O\left(N_{\varepsilon}^{\frac{34}{15}}\left(\beta_{\varepsilon}-1\right)^{\frac{4}{15}}\right)=o(1)
$$

thanks to (2.2). This proves that $\Phi_{\varepsilon} \rightarrow 0$ in $C^{1}\left(S^{6}\right)$ as $\varepsilon \rightarrow 0$, and thus that

$$
h_{\varepsilon} \rightarrow \Phi_{0} \text { in } C^{1}\left(S^{6}\right)
$$

as $\varepsilon \rightarrow 0$. Similar arguments give that $\Phi_{\varepsilon} \rightarrow 0$ in $C^{1}\left(S^{n}\right)$ when $n \geq 7$, and hence that $h_{\varepsilon} \rightarrow \Phi_{0}$ in $C^{1}\left(S^{n}\right)$ when $n \geq 7$. Now, in order to end the proof of Theorem 0.2 , there we still need to prove that the energy $E\left(u_{\varepsilon}\right)$ of $u_{\varepsilon}$ goes to $+\infty$ as $\varepsilon \rightarrow 0$. Noting that

$$
\int_{S^{n}} u_{\varepsilon}^{2^{\star}} d v_{h} \geq \sum_{i=1}^{N_{\varepsilon}} \int_{S^{n}} B_{i, \varepsilon}^{2^{\star}} d v_{h}
$$

and (see for instance Hebey [8]) that

$$
\int_{S^{n}} B_{i, \varepsilon}^{2^{\star}} d v_{h}=\left(\frac{n(n-2)}{4}\right)^{n / 2} \omega_{n}
$$

for all $i$ and all $\varepsilon>0$, where $\omega_{n}>0$ is the volume of $\left(S^{n}, h\right)$, we get that

$$
E\left(u_{\varepsilon}\right) \geq N_{\varepsilon}\left(\frac{n(n-2)}{4}\right)^{n / 2} \omega_{n} .
$$

In particular, $E\left(u_{\varepsilon}\right) \rightarrow+\infty$ as $\varepsilon \rightarrow 0$, and this ends the proof of Theorem 0.2.

\section{ACKNOWLEDGEMENTS}

This work was started while the authors were visiting Princeton University in November 2002. The authors thank this institution for its warm hospitality. The authors also express their deep thanks to Frédéric Robert for very useful comments on this work.

\section{REFERENCES}

[1] Brézis, H., and Coron, J.M., Convergence de solutions de $H$-systèmes et application aux surfaces à courbure moyenne constante, C. R. Acad. Sci. Paris, 298, 389-392, 1984. MR 0748929 (86d:35044)

[2] Convergence of solutions of $H$-systems or how to blow bubbles, Arch. Rational Mech. Anal., 89, 21-56, 1985. MR 0784102 (86g:53007)

[3] Caffarelli, L. A., Gidas, B., and Spruck, J., Asymptotic symmetry and local behavior of semilinear elliptic equations with critical Sobolev growth, Comm. Pure Appl. Math., 42, 271-297, 1989. MR 0982351 (90c:35075) 
[4] Druet, O., From one bubble to several bubbles. The low-dimensional case, J. Differential Geom., 63, 399-473, 2003. MR 2015469

[5] Druet, O., Compactness for Yamabe metrics in low dimensions, Internat. Math. Res. Notices, 23, 1143-1191, 2004. MR 2041549

[6] Druet, O., Hebey, E., and Robert, F., A $C^{0}$-theory for the blow-up of second order elliptic equations of critical Sobolev growth, Elect. Res. Ann. A.M.S, 9, 19-25, 2003. MR 1988868 (2004c:58046)

[7] _ Blow-up theory for elliptic PDEs in Riemannian geometry, Mathematical Notes, Princeton University Press, to appear.

[8] Hebey, E., Nonlinear analysis on manifolds: Sobolev spaces and inequalities, CIMS Lecture Notes, Courant Institute of Mathematical Sciences, Vol. 5, 1999. Second edition published by the American Mathematical Society, 2000. MR 1688256 (2000e:58011)

[9] _ Variational methods and elliptic equations in Riemannian geometry, Preprint, Notes from lectures given at ICTP, 2003. Available at http://www.u-cergy.fr/rech/pages/hebey.

[10] Li, Y.Y., and Zhang, L., A Harnack type inequality for the Yamabe equation in low dimensions, Calc. Var. Partial Differential Equations, to appear.

[11] Li, Y.Y, and Zhu, M., Yamabe type equations on three dimensional Riemannian manifolds, Communications in Contemporary Math., 1, 1-50, 1999. MR 1681811 (2000m:53051)

[12] Lions, P.-L., The concentration-compactness principle in the calculus of variations. The limit case I, II, Rev. Mat. Iberoamericana, no. 1, 145-201; no. 2, 45-121, 1985. MR 0834360 (87c:49007) MR 0850686 (87j:49012)

[13] Obata, M., The conjectures on conformal transformations of Riemannian manifolds, J. Differential Geom., 6, 247-258, 1971/72. MR 0303464 (46:2601)

[14] Sacks, P., and Uhlenbeck, K., On the existence of minimal immersions of 2-spheres, Ann. of Math., 113, 1-24, 1981. MR 0604040 (82f:58035)

[15] Schoen, R., Variational theory for the total scalar curvature functional for Riemannian metrics and related topics, Topics in calculus of variations (Montecatini Terme, 1987), 120-154. Lecture Notes in Mathematics, 1365, Springer, Berlin-New York, 1989. MR 0994021 (90g:58023)

[16] On the number of constant scalar curvature metrics in a conformal class, Differential Geometry: A symposium in honor of Manfredo Do Carmo, H.B.Lawson and K.Tenenblat eds., Pitman Monogr. Surveys Pure Appl. Math., 52, 311-320, 1991. MR 1173050 (94e:53035)

[17] Struwe, M., A global compactness result for elliptic boundary problems involving limiting nonlinearities, Math. Z., 187, 511-517, 1984. MR 0760051 (86k:35046)

[18] Wente, H.C., Large solutions to the volume constrained Plateau problem, Arch. Rational Mech. Anal., 75, 59-77, 1980. MR 0592104 (82a:49049)

Département de Mathématiques - UMPA, Ecole normale supérieure de Lyon, 46 allée D'Italie, 69364 Lyon Cedex 07, France

E-mail address: Olivier.Druet@umpa.ens-lyon.fr

Département de Mathématiques, Université de Cergy-Pontoise, Site de Saint-Martin, 2 avenue Adolphe Chauvin, 95302 Cergy-Pontoise cedex, France

E-mail address: Emmanuel.Hebey@math.u-cergy.fr 